\title{
Alzheimerschäden schon zehn Jahre vor der Diagnose
}

\section{Das frühe Erkennen von Prodromal- stadien der Alzheimer-Krankheit (AD) ist wichtig, weil neue Therapien nur während der frühen Phasen der Krankheit wirksam sind.}

— Wissenschaftler der Universität Lund in Malmö (Schweden) untersuchten in der Lumbalflüssigkeit die Biomarker Gesamt-Tau (T-Tau), phosphoryliertes Tau (P-Tau) und das $\beta$-Amyloid 1-42 (A 342$)$ über durchschnittlich 9,2 Jahre (4,1-11,8 Jahre) während der milden Entwicklung der $\mathrm{AD}$ und der frühen und späten $\mathrm{AD}$-Ausprägung.

$72(53,7 \%)$ der Patienten entwickelten eine AD und $21(15,7 \%)$ andere Demenzformen.
Die A 342 -Level waren im Vergleich zu den Gesunden reduziert und die TTau und P-Tau-Level erhöht $(\mathrm{p}<0,001)$. Die A $\beta 42 / P-T a u-R a t i o$ sagte die Entwicklung der AD mit einer Sensitivität von $88 \%$ und einer Spezifität von $90 \%$ voraus.

\footnotetext{
- Buchhave, P et al.

(Korrespondenzadresse: O. Hansson; Clinical Memory Research Unit, Dept. of Clinical Sci, Lund Univ Malmö, Sweden ; E-mail: Oskar Hansson@med.lund.se) Cerebrospinal fluid levels of $\beta$-amyloid 1-42, but not of tau, are fully changed already 5 to 10 years before the onset of Alzheimer dementia. Arch. Gen. Psychiatr. 2012; 69, 98-106; doi: 10.1001/archgenpsychiatr.2011155
}

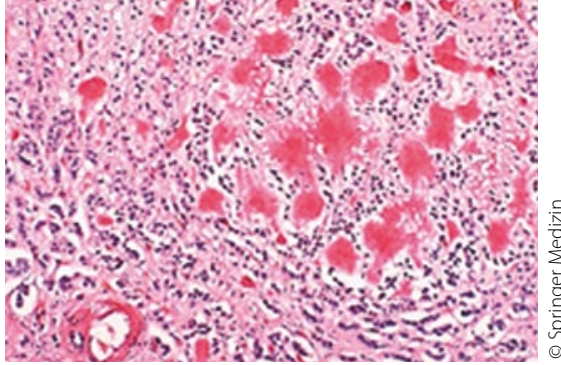

Amyloid-Plaques bei Alzheimer-Demenz.

\section{Kommentar}

Die ersten Gehirnveränderungen können bei Personen mit Alzheim-Demenz bereits zehn Jahre vor der klinischen Diagnose erfasst werden. Eine Sensitivität von $90 \%$ ist aber noch nicht auseichend. Die Kombination mit einem anderen Verfahren wie z. B. der MRI könnte sie erhöhen. Die Veränderungen scheinen mit denen des $\beta$-Amyloids zu beginnen, was wichtig für die Entwicklung neuer Therapien ist.

K. MALBERG =

\section{Achtung:}

\section{Hier muss der Dummy durch eine Anzeige ersetzt werden !!}

